\title{
Parameterized Regulator Synthesis for Bimodal Linear Systems Based on Bilinear Matrix Inequalities
}

\author{
Zhizheng Wu and Foued Ben Amara \\ Department of Mechanical and Industrial Engineering, University of Toronto, Toronto,
} ON, Canada M5S 3G8

Correspondence should be addressed to Foued Ben Amara, benamara@mie.utoronto.ca

Received 3 July 2007; Revised 18 January 2008; Accepted 7 March 2008

Recommended by Tamas Kalmar-Nagy

A regulator design method is presented for switched bimodal linear systems, where it is desired to reject known disturbance signals and/or track known reference inputs. The switching in the bimodal system is defined by a switching surface. The regulator design approach consists of three steps. The first step is based on constructing a switched observer-based state feedback central controller for the switched linear system. The second step involves augmenting the switched central controller with additional dynamics to construct a parameterized set of switched controllers. In the third step, two sufficient regulation conditions are derived for the resulting switched closed loop system. The regulation conditions present guidelines for the selection of the additional dynamics used to parameterize the switched controllers to yield the desired regulator. A regulator synthesis approach is proposed based on solving properly formulated bilinear matrix inequalities. Finally, a numerical example is presented to illustrate the performance of the proposed regulator.

Copyright (C) $2008 \mathrm{Z}$. Wu and F. Ben Amara. This is an open access article distributed under the Creative Commons Attribution License, which permits unrestricted use, distribution, and reproduction in any medium, provided the original work is properly cited.

\section{Introduction}

Recently, switched control systems have attracted much attention in the control community since they present challenging problems of practical importance. Significant progress has been made in this area, most notably in the stability analysis for such systems [1-7]. Numerous other results have been published and are presented in [8-10] and the references therein. In practice, in addition to stability requirements, there is a need to find controllers that would achieve regulation against known reference or disturbance signals. For example, in hard disk drives, maintaining a constant small distance between the read/write head and the disk surface is an important target that would allow greater data storage densities on the hard disk. However, the close proximity of the read/write head to the disk surface leads to intermittent contact 
between the two and results in a switched system regulation problem [11,12]. Solutions to the output regulation problem for the case of linear systems have been presented in [13-15] and for the case of nonlinear systems in $[16,17]$. However, these results cannot be applied to switched systems since the vector fields of switched systems are discontinuous and nonsmooth. The work of Devasia et al. [18] and Sakurama and Sugie [19] are closely related to this problem. Devasia et al. [18] studied the exact output tracking problem for linear switched systems and presented necessary and sufficient conditions for the existence of a regulator for exact output tracking with parameter jumps. The derived solution was based on the assumption that the switching times are known a priori. Therefore, the necessary and sufficient conditions were derived only for the elimination of switching induced output transients and cannot be applied to systems where switching is signal driven or where the switching times cannot be set a priori. Sakurama and Sugie [19] recently discussed the trajectory tracking problem for bimodal switched systems. The reference trajectory for the switched system is assumed known and the switching times are assumed to be measurable. An error variable and an error system are introduced, based on which the tracking controller was designed using a Lyapunov-like function. However, conditions for the existence of the desired regulator are sometimes very difficult to check.

In this paper, a controller design approach is proposed for single-input single-output switched bimodal systems, where it is desired to reject known disturbance signals and track known reference inputs simultaneously. Switching in the bimodal system is defined by a switching surface. A regulator synthesis method based on solving bilinear matrix inequalities (BMIs) is presented. The proposed regulator design approach consists of three steps. In the first step, a switched observer-based state-feedback central controller is constructed for the switched linear system. The second step involves augmenting the switched central controller with additional dynamics to construct a parameterized set of switched controllers. Stability analysis of the resulting switched closed loop system is then presented. In the third step, two sufficient regulation conditions are derived for the switched closed loop system. The first sufficient condition is derived based on the input-output stability property of the switched closed loop system. The second sufficient condition for regulation is derived by transforming the forced switched closed loop system into an unforced impulsive switched system. As such, the regulation problem is transformed into a stability analysis problem for the impulsive switched system. Based on the parameterized controller structure and the derived regulation conditions, proper BMIs are formulated and a regulator synthesis method is proposed. The main advantage of the proposed regulator synthesis approach is that it offers a numerical procedure that can practically be implemented and used to develop the desired switched regulator.

The rest of the paper is organized as follows. In Section 2, the general regulation problem for switched bimodal linear systems is presented. In Section 3, the construction of a parameterized set of switched controllers for the switched system is discussed and the stability properties of the resulting closed loop switched system are analyzed. Regulation conditions for the switched system are presented in Section 4 and the regulator synthesis method for switched systems is proposed in Section 5. The controller design method is illustrated in Section 6 using a numerical example, followed by the conclusion in Section 7 .

In the following, $\lambda_{\max }(\cdot)$ and $\lambda_{\min }(\cdot)$ denote the maximum and minimum eigenvalues of a matrix, respectively. The symbol $I$ represents the identity matrix. For a given $1 \times N$ matrix $X$, $\vec{X}$ denotes the $N \times 1$ vector with the same entries as those of $X^{T}$. 


\section{Regulation problem for switched bimodal systems}

This paper considers switched bimodal systems subject to external inputs representing disturbance and/or reference signals. Both the system dynamics as well as the external input signal are assumed to switch according to a switching law defined by a switching surface. These types of switched systems are motivated by practical applications, such as the flying height regulation problem for the read/write head in hard disk drives [11, 12].

Consider the switched system given by the following state space representation:

$$
\Sigma_{r}: \begin{cases}\dot{x}=A_{r} x+B_{r} u+D_{r}^{x} w_{r}, & x(0)=x_{0}, \\ \dot{w}_{r}=H_{r} w_{r}, & w_{r}(0)=w_{r 0}, \\ y=C_{r}^{y} x+D_{r}^{y} w_{r} & \\ e=C^{e} x+D_{r}^{e} w_{r,} & \\ r= \begin{cases}1 & \text { if } e=C^{e} x+D_{r}^{e} w_{r} \leq \delta, \\ 2 & \text { if } e=C^{e} x+D_{r}^{e} w_{r}>\delta,\end{cases} \end{cases}
$$

where $x \in \mathbb{R}^{n}$ is the state vector, $u \in \mathbb{R}$ is the control input, $y \in \mathbb{R}$ is the measurement signal to be fed to the controller, $e \in \mathbb{R}$ is the performance variable to be regulated and is assumed to be measurable, $w_{r} \in \mathbb{R}^{h}$ is the state vector of the exogenous systems generating the signal $w_{r}$, $H_{r} \in \mathbb{R}^{h \times h}$ have simple eigenvalues on the imaginary axis, $r \in\{1,2\}$ is the index of the system $\Sigma_{r}$ under consideration at time $t$, and $\delta$ is a constant satisfying $|\delta|>0$. The switching between the systems $\Sigma_{1}$ and $\Sigma_{2}$ is performed according to the value of the performance variable $e$, and is determined based on the location of $x$ with respect to a switching surface $S$ given by

$$
S=\left\{x \in \mathbb{R}^{n}: e=C^{e} x+D_{r}^{e} w_{r}=\delta\right\} .
$$

The switching surface $S$ is not fixed but changes with time given that the term $D_{r}^{e} w_{r}$ is in general a time-varying term. The switching between the two modes takes place as follows. If $r=1$ and $e$ becomes strictly greater than $\delta$, then the mode switches to $r=2$; and if $r=2$ and $e$ becomes less than or equal to $\delta$, then the mode switches to $r=1$. In the following, it is assumed that for any given $t \geq 0$, the system must operate in only one of the two modes corresponding to $r \in\{1,2\}$. This assumption is motivated by physical considerations in some applications of interest, such as the example presented at the end of the paper or the system treated in $[11,12]$. For the switched system (2.1), it is desired to construct an output feedback controller to regulate the performance variable $e$ of the switched system against the external input signal $w_{r}$. Given the switching nature of the plant, the output feedback controller is also chosen to be a switching feedback controller $\mathbb{C}_{r}, r \in\{1,2\}$, with a state space representation given by

$$
\mathbb{C}_{r}:\left\{\begin{array}{l}
\dot{x}_{c}=A_{r, c} x_{c}+B_{r, c} y, \\
u=C_{r, c} x_{c}
\end{array}\right.
$$


where $x_{c} \in \mathbb{R}^{n_{c}}$, and where the switching among controllers is to obey the same rule given in (2.1) for switching between the two plant models. Therefore, the resulting closed loop system is given by

$$
\Sigma_{r}^{\mathrm{cl}}:\left\{\begin{array}{l}
{\left[\begin{array}{c}
\dot{x} \\
\dot{x}_{c}
\end{array}\right]=\left[\begin{array}{cc}
A_{r} & B_{r} C_{r, c} \\
B_{r, c} C_{r}^{y} & A_{r, c}
\end{array}\right]\left[\begin{array}{l}
x \\
x_{c}
\end{array}\right]+\left[\begin{array}{c}
D_{r}^{x} \\
B_{r, c} D_{r}^{y}
\end{array}\right] w_{r},} \\
e=\left[\begin{array}{ll}
C^{e} & 0
\end{array}\right]\left[\begin{array}{l}
x \\
x_{c}
\end{array}\right]+D_{r}^{e} w_{r}, \\
r=\left\{\begin{array}{l}
1 \text { if } e=C^{e} x+D_{r}^{e} w_{r} \leq \delta, \\
2 \text { if } e=C^{e} x+D_{r}^{e} w_{r}>\delta .
\end{array}\right.
\end{array}\right.
$$

The output feedback regulation problem for the switched system (2.1) can then be stated as follows.

\section{Output-feedback regulation problem}

Given the switched system (2.1), find a switched output feedback controller of the form (2.3) such that the resulting closed loop system satisfies the following conditions.

(C1) Internal stability. With $w_{r}(0)=0$, the equilibrium point $\left[\begin{array}{ll}x^{T} & x_{c}^{T}\end{array}\right]^{T}=0$ of the unforced switched closed loop system is exponentially stable.

(C2) Output regulation. For all $\left[x^{T}(0) x_{c}^{T}(0)\right]^{T} \in \mathbb{R}^{n} \times \mathbb{R}^{n_{c}}$ and $w_{r}(0) \in \mathbb{R}^{h}$, the response $e(t)$ of the closed loop system involving the switched system (2.1) and the switched controller (2.3) satisfies

$$
\lim _{t \rightarrow \infty} e(t)=0 \text {. }
$$

In the following section, a framework within which regulation conditions will be derived is presented.

\section{Parameterization of a set of controllers}

The controller design approach presented in this paper relies on the construction of a parameterized set of output feedback controllers for the switched system (2.1). In this section, the construction of such a set is first discussed, followed by an analysis of the stability properties of the resulting switched closed loop system. The construction of a parameterized set of switched controllers involves two steps. The first step consists of designing a central controller in the form of an observer-based state-feedback controller. The second step involves augmenting the central controller with additional dynamics to construct a parameterized set of controllers.

\subsection{Observer-based state-feedback controller}

Consider the following observer-based state-feedback controller for the switched system (2.1)

$$
\mathbb{C}_{r}^{o}:\left\{\begin{array}{l}
\dot{\hat{x}}=A_{r} \hat{x}+B_{r} u+L_{r}(\widehat{y}-y), \quad \hat{x}(0)=\widehat{x}_{0}, \\
u=K_{r} \hat{x},
\end{array}\right.
$$




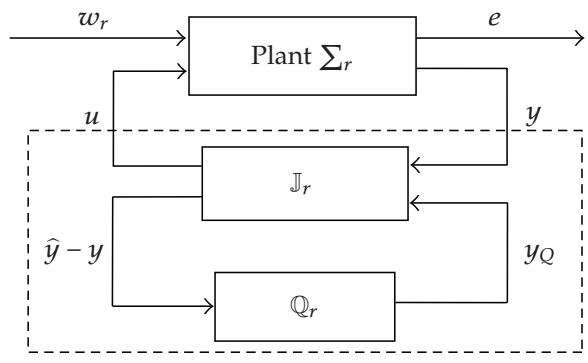

Figure 1: Closed loop system with a parameterized controller.

where $\widehat{x} \in \mathbb{R}^{n}$ is the estimate of the plant state vector $x$ and $\widehat{y}=C_{r}^{y} \widehat{x}$ is an estimate of the plant output $y$. The mode $r \in\{1,2\}$ is determined according to the rule given in (2.1). Moreover, it is assumed that there are no impulsive changes in the controller states at the switching times.

\subsection{Parameterized output-feedback controller}

The construction of a parameterized set of controllers for the switched system is based on considering, for each $r \in\{1,2\}$, a linear fractional transformation involving a fixed system $\mathbb{J}_{r}$ and a proper system $\mathbb{Q}_{r}$ as shown in Figure 1. The proposed controllers are similar to the parameterized stabilizing controllers for linear systems [20,21], but where no stability assumptions are placed on the system $\mathbb{Q}_{r}$. The state space representation of the system $\mathbb{J}_{r}$ is given by

$$
\mathbb{J}_{r}:\left\{\begin{array}{l}
\dot{\hat{x}}=\left(A_{r}+B_{r} K_{r}+L_{r} C_{r}^{y}\right) \widehat{x}-L_{r} y+\left[B_{r}\left(L_{r} D_{r}^{y}+D_{r}^{x}\right)\right] y_{Q}, \quad \widehat{x}(0)=\widehat{x}_{0}, \\
u=K_{r} \hat{x}+\left[\begin{array}{ll}
1 & 0 \\
1 \times q
\end{array}\right] y_{Q}, \\
\hat{y}-y=C_{r}^{y} \hat{x}-y,
\end{array}\right.
$$

and the system $\mathbb{Q}_{r}$ is given by

$$
\mathbb{Q}_{r}:\left\{\begin{array}{l}
\dot{x}_{Q}=A_{Q_{r}} x_{Q}+B_{Q_{r}}(\hat{y}-y), \quad x_{Q}(0)=x_{Q^{\prime}}^{0} \\
y_{Q}=\left[\begin{array}{c}
C_{Q_{r}} \\
I_{q \times q}
\end{array}\right] x_{Q}
\end{array}\right.
$$

where $x_{Q} \in \mathbb{R}^{q}$ and $y_{Q} \in \mathbb{R}^{q+1}$. In particular, throughout the rest of the paper, the system $\mathbb{Q}_{r}$ is such that the matrices $A_{Q_{r}}, B_{Q_{r}}$, and $C_{Q_{r}}$ also change according to $r$.

Remark 3.1. The state space representation of the system $\mathbb{J}_{r}$ given in (3.2) differs from that traditionally used in the construction of parameterized sets of stabilizing controllers for linear systems in that the state equation in (3.2) contains the additional term $\left(L_{r} D_{r}^{y}+D_{r}^{x}\right) x_{Q}$. The presence of this term makes it possible to derive the sufficient conditions for regulation presented in Theorem 4.2. 
By combining the systems $\mathbb{J}_{r}$ and $\mathbb{Q}_{r}$ in (3.2) and (3.3), the state-space representation of the regulator is then given by (2.3), where

$$
\begin{gathered}
x_{c}=\left[\begin{array}{c}
\hat{x} \\
x_{Q}
\end{array}\right], \quad A_{r, c}=\left[\begin{array}{cc}
A_{r}+B_{r} K_{r}+L_{r} C_{r}^{y} & B_{r} C_{Q_{r}}+L_{r} D_{r}^{y}+D_{r}^{x} \\
B_{Q_{r}} C_{r}^{y} & A_{Q_{r}}
\end{array}\right], \\
B_{r, c}=\left[\begin{array}{c}
-L_{r} \\
-B_{Q_{r}}
\end{array}\right], \quad C_{r, c}=\left[\begin{array}{ll}
K_{r} & C_{Q_{r}}
\end{array}\right], \quad D_{r, c}=0 .
\end{gathered}
$$

Let $\tilde{x}=\hat{x}-x$ denote the state estimation error. It follows that the resulting closed loop system involving the plant (2.1) and the regulator given by (2.3) and (3.4) can be written as follows:

$$
\Sigma_{r}^{\mathrm{cl}}:\left\{\begin{array}{l}
{\left[\begin{array}{c}
\dot{x} \\
\dot{\tilde{x}} \\
\dot{x}_{Q}
\end{array}\right]=\left[\begin{array}{ccc}
A_{r}+B_{r} K_{r} & B_{r} K_{r} & B_{r} C_{Q_{r}} \\
0 & A_{r}+L_{r} C_{r}^{y} & D_{r}^{x}+L_{r} D_{r}^{y} \\
0 & B_{Q_{r}} C_{r}^{y} & A_{Q_{r}}
\end{array}\right]\left[\begin{array}{c}
x \\
\tilde{x} \\
x_{Q}
\end{array}\right]+\left[\begin{array}{c}
D_{r}^{x} \\
-D_{r}^{x}-L_{r} D_{r}^{y} \\
-B_{Q_{r}} D_{r}^{y}
\end{array}\right] w_{r},} \\
e=\left[\begin{array}{lll}
C^{e} & 0 & 0
\end{array}\right]\left[\begin{array}{c}
x \\
\tilde{x} \\
x_{Q}
\end{array}\right]+D_{r}^{e} w_{r} .
\end{array}\right.
$$

Let $\mathcal{X}=\left[\begin{array}{lll}x^{T} & \tilde{x}^{T} & x_{Q}^{T}\end{array}\right]_{1 \times N}^{T}$ denote the state vector of the resulting closed loop system with $N=$ $2 n+q$, and let

$$
\begin{gathered}
\widehat{A}_{r}=\left[\begin{array}{ccc}
A_{r}+B_{r} K_{r} & B_{r} K_{r} & B_{r} C_{Q_{r}} \\
0 & A_{r}+L_{r} C_{r}^{y} & D_{r}^{x}+L_{r} D_{r}^{y} \\
0 & B_{Q_{r}} C_{r}^{y} & A_{Q_{r}}
\end{array}\right], \\
E_{r}=\left[\left(D_{r}^{x}\right)^{T}-\left(D_{r}^{x}+L_{r} D_{r}^{y}\right)^{T}-\left(B_{Q_{r}} D_{r}^{y}\right)^{T}\right]^{T}, \quad C^{e x}=\left[\begin{array}{lll}
C^{e} & 0 & 0
\end{array}\right] .
\end{gathered}
$$

The resulting parameterized switched closed loop system dynamics can then be expressed as

$$
\Sigma_{r}^{\mathrm{cl}}:\left\{\begin{array}{l}
\dot{x}=\widehat{A}_{r} X+E_{r} w_{r}, \\
e=C^{e x} X+D_{r}^{e} w_{r}, \\
r=\left\{\begin{array}{l}
1 \text { if } e=C^{e x} X+D_{r}^{e} w_{r} \leq \delta, \\
2 \text { if } e=C^{e x} X+D_{r}^{e} w_{r}>\delta .
\end{array}\right.
\end{array}\right.
$$

In the following, both the internal stability as well as the input-output stability properties of the system (3.7) are analyzed. 


\subsection{Stability of the parameterized switched closed loop system}

In this section, it is desired to study the stability properties of the system (3.7). The stability analysis involves two steps, namely, internal stability analysis and input-output stability analysis. In the first step, the internal stability of the closed loop system is studied by considering the system (3.7) in the absence of the signal $w_{r}$, and studying the stability properties of the origin for the resulting unforced switched system. The second step builds on the internal stability results and presents input-output stability results for the system (3.7).

\subsubsection{Internal stability}

Consider first the system (3.7) in the absence of the signal $w_{r}$. The state equation for the resulting system is given by

$$
\dot{X}=\widehat{A}_{r} X
$$

Note that, there are no impulsive changes in the state variables of the above switched system at the switching times. The internal stability of the system (3.8) is then given by the following well known result.

Lemma 3.2 (see [22]). If there exists a matrix $P=P^{T}>0$ and a constant $\alpha$ such that the following inequalities hold

$$
\widehat{A}_{r}^{T} P+P \widehat{A}_{r}+2 \alpha P<0, \quad \alpha>0, r \in\{1,2\},
$$

then the origin is an exponentially stable equilibrium point for the switched system (3.8) with arbitrary switching, and

$$
\|\chi(t)\|<c e^{-\alpha t}\|\chi(0)\|,
$$

where $c=\sqrt{\lambda_{\max }(P) / \lambda_{\min }(P)}$.

Remark 3.3. The matrix inequality (3.9) can be solved using numerical algorithms. Here, we search for a common Lyapunov function for the switched systems (3.8) to guarantee the stability of the origin for the system (3.8) under arbitrary switching, since the switching surface $S=\left\{x \in \mathbb{R}^{n}: e=C^{e} x+D_{r}^{e} w_{r}=\delta\right\}$ is not a fixed function of the state vector $x$ and changes with $D_{r}^{e} w_{r}$.

\subsubsection{Input-output stability}

In this section, we will consider the input-output stability properties of the system (3.7) with the external input signal $w_{r}$. Let $w_{m}=\max _{r \in\{1,2\} ; t \geq 0}\left\|E_{r} w_{r}(t)\right\|$. Then we have the following stability result.

Lemma 3.4 (see [9]). Assume the origin for the switched system (3.8) is an exponentially stable equilibrium point. Then the state vector in (3.7) is bounded, and the states will ultimately evolve inside the bounded set $B_{\beta}$ given by

$$
B_{\beta}=\left\{x \in \mathbb{R}^{N}:\|x\| \leq \beta\right\},
$$

where $\beta=c w_{m} / \alpha$.

The input-output stability of the closed loop system follows immediately from the above result. 


\section{Regulation conditions for the switched system}

The purpose of introducing the parameterized controllers is to find, for each $r \in\{1,2\}$, appropriate $A_{Q_{r}}, B_{Q_{r}}$, and $C_{Q_{r}}$ in (3.3) to solve the output-feedback regulation problem for the switched system (3.7). The solution to the regulation problem for the switched system (3.7) is presented in two steps. In the first step, regulation conditions for each of the individual closed loop systems $\Sigma_{r}^{\mathrm{cl}}, r \in\{1,2\}$, are presented. In the second step, two sufficient conditions for regulation in the switched closed loop systems $\sum_{r}^{\mathrm{cl}}$ are derived. To derive the sufficient condition for regulation, a coordinate transformation is defined first, allowing the forced switched closed loop system to be transformed into an unforced impulsive switched system. Hence, the regulation problem is transformed into a stability analysis problem for the origin of the resulting impulsive switched system. Conditions for achieving asymptotic stability in the new impulsive switched system are then presented, which is equivalent to achieving regulation in the original switched closed loop system.

\subsection{Regulation conditions for $\Sigma_{1}^{\mathrm{cl}}$ and $\Sigma_{2}^{\mathrm{cl}}$}

Let $\bar{A}_{r}=\left[\begin{array}{cc}A_{r} & B_{r} C_{r, c} \\ B_{r, c} C_{r}^{y} & A_{r, c}\end{array}\right]$ and $\bar{E}_{r}=\left[\begin{array}{c}D_{r}^{x} \\ B_{r, c} D_{r}^{y}\end{array}\right]$ in (2.4). For each of the systems $\Sigma_{r}, r \in\{1,2\}$, and using a controller of the form (2.3), regulation conditions are given by the following lemma.

Lemma 4.1 (see [15]). For each $r \in\{1,2\}$, consider the system $\Sigma_{r}$ in (2.1) and a controller $\mathbb{C}_{r}$ in (2.3), and assume that $\bar{A}_{r}$ is a stability matrix. Then, for each $r \in\{1,2\}, \Sigma_{r}^{\mathrm{cl}}$ in (2.4) achieves regulation if and only if there exists a matrix $\Pi_{r}$ that solves the linear matrix equations:

$$
\begin{gathered}
\Pi_{r} H_{r}=\bar{A}_{r} \Pi_{r}+\bar{E}_{r}, \\
0=C^{e x} \Pi_{r}+D_{r}^{e} .
\end{gathered}
$$

Similarly, using the parameterized feedback regulator $\mathbb{C}_{r}$ given in (2.3) and (3.4), regulation conditions for the switched closed loop system (3.7) can be derived and are presented in the following theorem.

Theorem 4.2. Assume that for each $r \in\{1,2\}$, the closed loop system $\Sigma_{r}^{\mathrm{cl}}$ given in (3.7) is exponentially stable. Then each of the systems $\Sigma_{1}^{\mathrm{cl}}$ and $\Sigma_{2}^{\mathrm{cl}}$ achieves regulation only if, for each $r \in\{1,2\}$, there exists a pair of matrices $\left(\Omega_{r}, \Psi_{r}\right)$ which satisfy the following equations:

$$
\begin{aligned}
\Omega_{r} H_{r} & =A_{r} \Omega_{r}+B_{r} \Psi_{r}+D_{r}^{x}, \\
0 & =C^{e} \Omega_{r}+D_{r}^{e} .
\end{aligned}
$$

Furthermore, if the linear matrix equations in (4.2) admit a solution $\left(\Omega_{r}, \Psi_{r}\right)$, and the matrices $A_{Q_{r}}$ and $C_{Q_{r}}$ in (3.3) are taken to be of the form

$$
\begin{gathered}
A_{Q_{r}}=H_{r}+B_{Q_{r}} D_{r}^{y}, \\
C_{Q_{r}}=\Psi_{r}-K_{r} \Omega_{r},
\end{gathered}
$$

then, for each $r \in\{1,2\}, \Sigma_{r}^{\mathrm{cl}}$ in (3.7) achieves regulation. 
Proof. Equation (4.1) can be rewritten as

$$
\begin{aligned}
\Pi_{r} H_{r} & =\left[\begin{array}{cc}
A_{r} & B_{r} C_{r, c} \\
B_{r, c} C_{r}^{y} & A_{r, c}
\end{array}\right] \Pi_{r}+\left[\begin{array}{c}
D_{r}^{x} \\
B_{r, c} D_{r}^{y}
\end{array}\right], \\
0 & =\left[\begin{array}{ll}
C^{e} & 0
\end{array}\right] \Pi_{r}+D_{r}^{e} .
\end{aligned}
$$

Partition $\Pi_{r}$ as $\Pi_{r}=\left[\begin{array}{c}\Omega_{r} \\ \Phi_{r}\end{array}\right]$. Then (4.1) is the same as

$$
\begin{gathered}
\Omega_{r} H_{r}=A_{r} \Omega_{r}+B_{r} C_{r, c} \Phi_{r}+D_{r}^{x}, \\
\Phi_{r} H_{r}=A_{r, c} \Phi_{r}+B_{r, c}\left(C_{r}^{y} \Omega_{r}+D_{r}^{y}\right), \\
0=C^{e} \Omega_{r}+D_{r}^{e} .
\end{gathered}
$$

Letting $\Psi_{r}=C_{r, c} \Phi_{r}$, (4.2) follows immediately.

To prove the second part of the theorem, note that by assumption, for each $r \in\{1,2\}, \widehat{A}_{r}$ in (3.7) is a stability matrix. Moreover, since $\bar{A}_{r}$ is related to $\widehat{A}_{r}$ by a similarity transformation, then $\bar{A}_{r}$ is also a stability matrix. Now suppose $\Omega_{r}$ and $\Psi_{r}$ satisfy (4.2) and that $A_{Q_{r}}$ and $C_{Q_{r}}$ satisfy (4.3). To show that the closed loop system achieves regulation, let $\Phi_{r}=\left[\begin{array}{c}\Omega_{r} \\ I\end{array}\right]$. Using the expression for $A_{r, c}$ in (3.4) and substituting in the expression for $A_{Q_{r}}$ in (4.3) yields

$$
A_{r, c}=\left[\begin{array}{cc}
A_{r}+B_{r} K_{r}+L_{r} C_{r}^{y} & B_{r} C_{Q_{r}}+L_{r} D_{r}^{y}+D_{r}^{x} \\
B_{Q_{r}} C_{r}^{y} & H_{r}+B_{Q_{r}} D_{r}^{y}
\end{array}\right]=\left[\begin{array}{cc}
A_{r} & D_{r}^{x} \\
0 & H_{r}
\end{array}\right]+\left[\begin{array}{c}
B_{r} \\
0
\end{array}\right]\left[\begin{array}{ll}
K_{r} & C_{Q_{r}}
\end{array}\right]+\left[\begin{array}{c}
L_{r} \\
B_{Q_{r}}
\end{array}\right]\left[\begin{array}{ll}
C_{r}^{y} & D_{r}^{y}
\end{array}\right] .
$$

It follows that:

$$
\begin{aligned}
A_{r, c} \Phi_{r} & =\left[\begin{array}{c}
A_{r} \Omega_{r}+D_{r}^{x} \\
H_{r}
\end{array}\right]+\left[\begin{array}{c}
B_{r} K_{r} \Omega_{r}+B_{r} C_{Q_{r}} \\
0
\end{array}\right]+\left[\begin{array}{c}
L_{r} \\
B_{Q_{r}}
\end{array}\right]\left(C_{r}^{y} \Omega_{r}+D_{r}^{y}\right) \\
& =\left[\begin{array}{c}
A_{r} \Omega_{r}+B_{r}\left(K_{r} \Omega_{r}+C_{Q_{r}}\right)+D_{r}^{x} \\
H_{r}
\end{array}\right]-B_{r, c}\left(C_{r}^{y} \Omega_{r}+D_{r}^{y}\right) .
\end{aligned}
$$

Given that $\Psi_{r}=C_{Q_{r}}+K_{r} \Omega_{r}$ from (4.3), then based on (4.2) we have

$$
A_{r, c} \Phi_{r}=\left[\begin{array}{c}
A_{r} \Omega_{r}+B_{r} \Psi_{r}+D_{r}^{x} \\
H_{r}
\end{array}\right]-B_{r, c}\left(C_{r}^{y} \Omega_{r}+D_{r}^{y}\right)=\Phi_{r} H_{r}-B_{r, c}\left(C_{r}^{y} \Omega_{r}+D_{r}^{y}\right)
$$

Therefore, (4.5) is satisfied, which implies (4.1) is satisfied and regulation can be achieved.

The results presented above represent regulation conditions for each of the closed loop systems in (3.7), individually. In the following sections, regulation conditions for the system (3.7) subject to switching are discussed. 


\subsection{Equivalent impulsive switched closed loop system model}

Based on Lemma 4.1, and using a properly defined coordinate transformation, the forced switched closed loop system (3.7) can be transformed into an unforced impulsive switched system. To introduce the appropriate coordinate transformation, note that the original regulation condition (4.1) can be rewritten as

$$
\begin{gathered}
\widehat{\Pi}_{r} H_{r}=\widehat{A}_{r} \widehat{\Pi}_{r}+E_{r}, \\
0=C^{e x} \widehat{\Pi}_{r}+D_{r}^{e},
\end{gathered}
$$

where

$$
\widehat{\Pi}_{r}=\left[\begin{array}{ccc}
I & 0 & 0 \\
-I & I & 0 \\
0 & 0 & I
\end{array}\right] \Pi_{r}
$$

Consider now the following coordinate transformation

$$
\tilde{x}=x-\widehat{\Pi}_{r} w_{r} .
$$

Using (4.9), the forced closed loop switched system (3.7) can be transformed into an unforced switched system given by

$$
\tilde{\Sigma}_{r}^{\mathrm{cl}}:\left\{\begin{array}{l}
\dot{\tilde{X}}=\widehat{A}_{r} \tilde{X}^{2} \\
e=C^{e x} \tilde{X}, \\
r=\left\{\begin{array}{l}
1 \text { if } e=C^{e x} \tilde{X} \leq \delta, \\
2 \text { if } e=C^{e x} \tilde{x}>\delta .
\end{array}\right.
\end{array}\right.
$$

It should be noted from (4.11) that the coordinate transformation varies depending on the value of $r \in\{1,2\}$. Consequently, the states $\tilde{X}(t)$ in the new system (4.12) undergo impulsive changes at the switching times. Let $\mathbb{T}_{s}$ be the set of switching times $\tau_{k}, k=1,2, \ldots$. Therefore, for a given switching time $\tau_{k} \in \mathbb{T}_{s}$, we have that for $t=\tau_{k}$

$$
\tilde{x}\left(t^{+}\right)=\tilde{x}\left(t^{-}\right)+\Delta \tilde{x}(t),
$$

where $\Delta \tilde{X}(t)=\widehat{\Pi}_{r\left(t^{-}\right)} w_{r\left(t^{-}\right)}(t)-\widehat{\Pi}_{r\left(t^{+}\right)} w_{r\left(t^{+}\right)}(t)$. Hence, the impulsive switched closed loop system is given by

$$
\tilde{\Sigma}_{r}^{\mathrm{cl}}:\left\{\begin{array}{l}
\dot{\tilde{X}}=\widehat{A}_{r} \tilde{X}, \\
\tilde{X}\left(t^{+}\right)=\tilde{X}\left(t^{-}\right)+\Delta \tilde{X}(t), \quad t \in \mathbb{T}_{s}, \\
e=C^{e x} \tilde{X}, \\
r=\left\{\begin{array}{l}
1 \text { if } e=C^{e x} \tilde{X} \leq \delta, \\
2 \text { if } e=C^{e x} \tilde{X}>\delta .
\end{array}\right.
\end{array}\right.
$$


In the new coordinate system, the switching surface $S$ given in (2.2) is fixed and is expressed as follows:

$$
S=\left\{\tilde{x} \in \mathbb{R}^{N}: e=C^{e x} \tilde{x}=\delta\right\}
$$

Using the coordinate transformation in (4.11), the original output-feedback regulation problem for the switched system (3.7) is transformed into an asymptotic stability analysis problem for the origin of the unforced impulsive switched system (4.14).

\subsection{Regulation conditions for the switched system}

First, let $\delta_{0}$ denote the distance between the switching surface $S$ and the origin. Then we have $\delta_{0}=|\delta| /\left\|C_{e x}\right\|$. Define

$$
\Delta \beta_{m}=\max _{\substack{r \in\{1,2\} \\ t \geq 0}}\left\|\widehat{\Pi}_{r} w_{r}(t)\right\|
$$

Let $\gamma=\beta+\Delta \beta_{m}+\varepsilon$, where $\varepsilon \ll 1$, and define the set $B_{\gamma}$ as follows:

$$
B_{\gamma}=\left\{\tilde{x} \in \mathbb{R}^{N}:\|\tilde{x}\| \leq r\right\} .
$$

Using the definition for $\tilde{X}$ in (4.11), and the fact that the original state vector $\chi$ will ultimately evolve inside a bounded set $B_{\beta}$ given by (3.11), it follows that there exists a finite time $T_{\gamma}$ at which $\tilde{X}$ will enter the set $B_{\gamma}$ and continue to evolve in $B_{\gamma}$ thereafter. A sufficient condition for regulation is then given as follows.

Theorem 4.3. Assume that the switched closed loop system $\sum_{r}^{\mathrm{cl}}$ given in (3.7) is internally stable under arbitrary switching, and that for each $r \in\{1,2\}, \Sigma_{r}^{\mathrm{cl}}$ given in (3.7) achieves output regulation. If

$$
\delta_{0}>r
$$

then the origin is an asymptotically stable equilibrium point for the impulsive switched system (4.14), implying that the switched system (3.7) achieves regulation.

Proof. If $\delta_{0}>\gamma$, then the switching surface $S$ given by (4.15) does not intersect the bounded set $B_{\gamma}$. The trajectory of the state vector $\tilde{X}$ will enter the set $B_{\gamma}$ at $t=T_{\gamma}$ and continue to evolve inside $B_{\gamma}$ thereafter. Consequently, there will be no more switching. Since the system $\Sigma_{r}^{\mathrm{cl}}$ that is active in the halfspace containing $B_{\gamma}$ is such that $\widehat{A}_{r}$ is a stability matrix, and since $0 \in B_{\gamma}$, the trajectory of the state vector $\tilde{X}$ asymptotically converges to the origin, implying that regulation in the original switched closed loop system (3.7) is achieved.

If condition (4.18) is not satisfied, that is, $\delta_{0} \leq \gamma$, then the switching surface $S$ must intersect with the bounded set $B_{\gamma}$. To present regulation conditions in this case, define the matrices $A_{s 1}=\left[\begin{array}{c}C^{e x} \\ C^{e x} \widehat{A}_{1}\end{array}\right], A_{s 2}=\left[\begin{array}{c}C^{e x} \\ C^{e x} \widehat{A}_{2}\end{array}\right]$, and $b=\left[\begin{array}{l}\delta \\ 0\end{array}\right]$. Sufficient conditions for regulation are presented in the following theorem. 
Theorem 4.4. Assume the switched closed loop system $\sum_{r}^{\mathrm{cl}}$ given in (3.7) is internally stable under arbitrary switching and that, for each $r \in\{1,2\}, \Sigma_{r}^{\mathrm{cl}}$ given in (3.7) achieves output regulation. Moreover, assume that $\delta_{0} \leq \gamma, \operatorname{rank}\left(A_{s 1}\right)=\operatorname{rank}\left(A_{s 2}\right)=2$, and that the direction of vector $\overrightarrow{C^{e x}}$ is not parallel to that of $\overrightarrow{C^{e x} \vec{A}_{1}}$ and $\overrightarrow{C^{e x} \widehat{A}_{2}}$. If the following conditions are satisfied,

$$
\begin{gathered}
C^{e x}\left(C^{e x} \widehat{A}_{r}\right)^{T}<0, \\
b^{T}\left(A_{s r} A_{s r}^{T}\right)^{-1} b>r^{2}, \\
r=1 \quad \text { if } \delta>0, \\
r=2 \quad \text { if } \delta<0,
\end{gathered}
$$

or

$$
\begin{gathered}
C^{e x}\left(C^{e x} \widehat{A}_{r}\right)^{T}>0, \\
b^{T}\left(A_{s r} A_{s r}^{T}\right)^{-1} b>r^{2}, \\
r=2 \quad \text { if } \delta>0, \\
r=1 \quad \text { if } \delta<0,
\end{gathered}
$$

then the origin is an asymptotically stable equilibrium point for the impulsive switched system (4.14), implying that the switched system (3.7) achieves regulation.

Proof. The following proof is presented for the case of $\delta>0$. The case of $\delta<0$ is treated using the same ideas. Let region 1 and region 2 denote the halfspaces where the systems $\widetilde{\Sigma}_{1}^{\mathrm{cl}}$ and $\widetilde{\Sigma}_{2}^{\mathrm{cl}}$ are active, respectively (see Figures 2 and 3 ). Hence, the origin is in region 1 where the system $\widetilde{\Sigma}_{1}^{\mathrm{cl}}$ is active. Define the hypersurfaces $S_{v 1}=\left\{\tilde{X} \in \mathbb{R}^{N}: C^{e x} \widehat{A}_{1} \tilde{X}=0\right\}$ and $S_{v 2}=$ $\left\{\tilde{\chi} \in \mathbb{R}^{N}: C^{e x} \widehat{A}_{2} \tilde{X}=0\right\}$. Then for all points in $S_{v 1}, \dot{e}=C^{e x} \widehat{A}_{1} \tilde{X}=0$ and for all points in $S_{v 2}$, $\dot{e}=C^{e x} \widehat{A}_{2} \tilde{X}=0$. Since, by assumption, $\delta_{0} \leq \gamma$, the switching surface $S$ intersects with the bounded set $B_{\gamma}$. Let $S_{\gamma}=\left\{\tilde{x} \in \mathbb{R}^{N}: C^{e x} \tilde{x}=\delta\right.$ and $\left.\|\tilde{x}\| \leq \gamma\right\}$ be the intersection of the switching surface $S$ with $B_{\gamma}$. By assumption, the direction of vector $\overrightarrow{C^{e x}}$ is not parallel to that of $\overrightarrow{C^{e x} \vec{A}_{1}}$ and $\overrightarrow{C^{e x} \widehat{A}_{2}}$. Therefore, the switching surface $S$ must intersect with either of the hypersurfaces $S_{v 1}$ or $S_{v 2}$. Define the intersection of $S$ with the hypersurfaces $S_{v 1}$ and $S_{v 2}$ as $L_{1}=\left\{\tilde{x} \in \mathbb{R}^{N}\right.$ : $C^{e x} \widehat{A}_{1} \tilde{x}=0$ and $\left.C^{e x} \tilde{x}=\delta\right\}$ and $L_{2}=\left\{\tilde{x} \in \mathbb{R}^{N}: C^{e x} \widehat{A}_{2} \tilde{x}=0\right.$ and $\left.C^{e x} \tilde{x}=\delta\right\}$, respectively. For each $r \in\{1,2\}$, let $l_{r}=\min _{\tilde{x} \in L_{r}}\|\tilde{x}\|$ denote the distance between the origin and the set $L_{r}$. Then, $l_{r}$ can be obtained by solving the problem of minimizing $\|\tilde{x}\|$ subject to the constraint $A_{s r} \tilde{X}=b$. Since, by assumption, $\operatorname{rank}\left(A_{s 1}\right)=\operatorname{rank}\left(A_{s 2}\right)=2$, the solution to the above problem is $l_{r}=\sqrt{b^{T}\left(A_{s r} A_{s r}^{T}\right)^{-1} b}, r \in\{1,2\}$. If $l_{r}^{2}=b^{T}\left(A_{s r} A_{s r}^{T}\right)^{-1} b>r^{2}$, then the sets $L_{r}, r \in\{1,2\}$, are outside the bounded set $B_{\gamma}$. In this situation, the set $S_{\gamma}$ does not intersect with the sets $S_{v 1}$ or $S_{v 2}$. If $C^{e x}\left(C^{e x} \widehat{A}_{1}\right)^{T}<0$ and $l_{1}^{2}=b^{T}\left(A_{s 1} A_{s 1}^{T}\right)^{-1} b>\gamma^{2}$, then the set $S_{\gamma}$ will be located in one of the two halfspaces defined by the hypersurface $S_{v 1}$ and where all the points satisfy $\dot{e}=C^{e x} \widehat{A}_{1} \tilde{X}<0$. Similarly, if $C^{e x}\left(C^{e x} \widehat{A}_{2}\right)^{T}>0$ and $l_{2}^{2}=b^{T}\left(A_{s 2} A_{s 2}^{T}\right)^{-1} b>\gamma^{2}$, then the set $S_{\gamma}$ will be located in one of the two halfspaces defined by the hypersurface $S_{v 2}$ and where all the points satisfy $\dot{e}=C^{e x} \widehat{A}_{2} \tilde{X}>0$. By deriving conditions on $\dot{e}$, and using the stability properties of $\widetilde{\Sigma}_{1}^{\mathrm{cl}}$ 


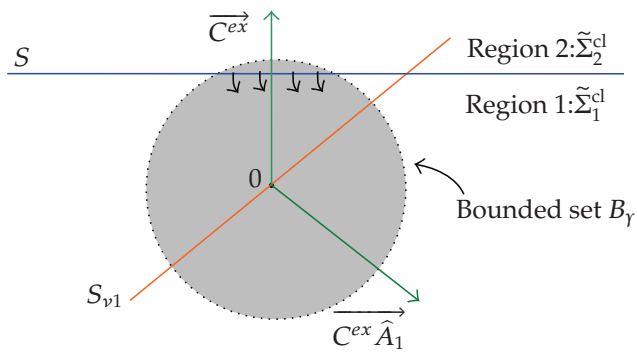

Figure 2: State trajectories of $\widetilde{\Sigma}_{1}^{\mathrm{cl}}$ with initial conditions on the switching surface inside the set $B_{\gamma}$ for Case 1.

and $\widetilde{\Sigma}_{2}^{\mathrm{cl}}$, it is possible to conclude about the asymptotic stability of the origin of the impulsive switched closed loop system (4.14). In the following, two cases are considered in detail.

Case $1\left(C^{e x}\left(C^{e x} \widehat{A}_{1}\right)^{T}<0\right.$ and $b^{T}\left(A_{s 1} A_{s 1}^{T}\right)^{-1} b>\gamma^{2}$ (see Figure 2$)$ ). In this case, all the state trajectories leaving the set $S_{\gamma}$ and entering region 1 have $\dot{e}<0$. Therefore, if the state trajectory leaves the set $S_{\gamma}$ and enters region 1, then it will not hit it again. Two possible cases can be considered here depending on whether the state trajectory enters the bounded set $B_{\gamma}$ from region 1 or region 2 at time $T_{\gamma}$. In this case, the state trajectory enters the set $B_{\gamma}$ from region 1, then the state trajectory cannot hit the switching surface $S$ since $\dot{e}<0$ for all states on the switching surface within the set $B_{\gamma}$. Therefore, the state trajectory is confined to evolve in region 1 inside the set $B_{\gamma}$. Since $\tilde{\Sigma}_{1}^{\mathrm{cl}}$ is a stable system, the state trajectory will converge to the origin asymptotically, which implies that regulation for the switched system (3.7) is achieved. Consider now the case where the state trajectory enters the set $B_{\gamma}$ from region 2. Since the state trajectory is confined to evolve inside the set $B_{\gamma}$ and since the system $\widetilde{\Sigma}_{2}^{\mathrm{cl}}$ is asymptotically stable, the state trajectory must hit the switching surface and switching must take place. Following switching, and based on the dynamics of the impulsive switched closed system as presented in (4.14), the state vector immediately following switching will be in region 1 . Since $\dot{e}<0$ for all states on the switching surface within the set $B_{\gamma}$ for the system $\widetilde{\Sigma}_{1}^{\mathrm{cl}}$, the state trajectory cannot cross the switching surface again and will continue to evolve in region 1 thereafter. The state trajectory will approach the origin asymptotically, which implies that regulation will be achieved.

Case $2\left(C^{e x}\left(C^{e x} \widehat{A}_{2}\right)^{T}>0\right.$ and $b^{T}\left(A_{s 2} A_{s 2}^{T}\right)^{-1} b>\gamma^{2}$ (see Figure 3$)$ ). In this case, all the state trajectories leaving the set $S_{\gamma}$ and entering region 2 have $\dot{e}>0$. Assume the state trajectory enters the set $B_{\gamma}$ from region 2 at the time $T_{\gamma}$. Given that the system $\widetilde{\Sigma}_{2}^{\mathrm{cl}}$ is asymptotically stable, and that the trajectories are confined to evolve inside $B_{\gamma}$, the state trajectory must cross the switching surface to approach the origin. However, this is not possible since, with respect to the system $\widetilde{\Sigma}_{2}^{\mathrm{cl}}, \dot{e}>0$ for all $\tilde{X} \in S_{\gamma}$. Therefore, it is not possible to have the state trajectory enter the set $B_{\gamma}$ from region 2 at time $T_{\gamma}$. Consider now the case, where the state trajectory enters the set $B_{\gamma}$ from region 1 . If the state trajectory crosses the switching surface to enter into region 2, then that will result in a contradiction similar to that discussed for the case, where the state trajectory enters the set $B_{\gamma}$ from region 2 . Therefore, once the state trajectory enters the set $B_{\gamma}$ from region 1, it will continue to evolve in region 1 forever and will never cross the switching surface to enter into region 2 for $t>T_{\gamma}$. Since $\widetilde{\Sigma}_{1}^{\mathrm{cl}}$ is a stable system, then the state trajectory will converge to the origin asymptotically, which implies that regulation for the switched system (3.7) is achieved. 


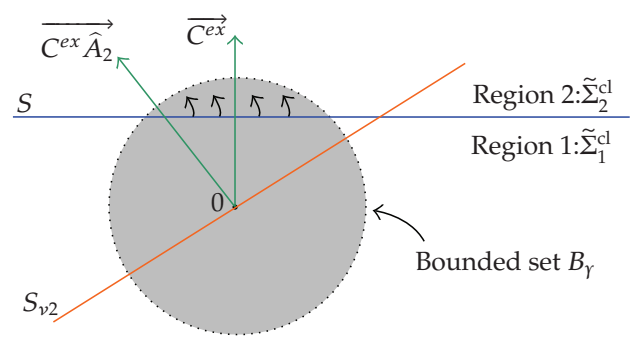

Figure 3: State trajectories of $\widetilde{\Sigma}_{2}^{\mathrm{cl}}$ with initial conditions on the switching surface inside the set $B_{\gamma}$ for Case 2.

Therefore, based on the above analysis, if the conditions for Case 1 given by (4.19) or the conditions for Case 2 given by (4.20) are satisfied, then regulation in the switched system (3.7) can be achieved.

\section{Regulator synthesis for the switched system}

Based on the regulation conditions for the switched system proposed in the previous section, a regulator synthesis approach is presented in this section. The proposed synthesis approach is based on solving a set of properly formulated bilinear matrix inequalities (BMIs). The main idea behind the regulator synthesis approach is as follows. Consider an output-feedback controller $\mathbb{C}_{r}$ as given in (2.3) and (3.4), where $A_{Q_{r}}=H_{r}+B_{Q_{r}} D_{r}^{y}$ and $C_{Q_{r}}=\Psi_{r}-K_{r} \Omega_{r}$. Since $\left(\Omega_{r}, \Psi_{r}\right)$ is the solution to the Sylvester equation (4.2), $\mathbb{C}_{r}$ is only parameterized in the unknown matrices $K_{r}, L_{r}$, and $B_{Q_{r}}$. Assume $\mathbb{C}_{r}$ is such that the resulting closed system satisfies (3.9). Then, based on Lemma 3.2 and Theorem 4.2, the switched closed loop system $\Sigma_{r}^{\mathrm{cl}}$ given in (3.7) is internally stable under arbitrary switching and, for each $r \in\{1,2\}, \Sigma_{r}^{\mathrm{cl}}$ given in (3.7) also achieves output regulation. In this case, if any of (4.18), (4.19), and (4.20) is satisfied, then based on Theorems 4.3 and 4.4, regulation in the switched system (3.7) is achieved. The set of conditions in Lemma 3.2 and Theorems 4.3 and 4.4 yield bilinear matrix inequalities in the unknown parameters $K_{r}, L_{r}$, and $B_{Q_{r}}$. In the following, a solution procedure for the formulated BMIs is proposed to determine the unknown parameters $K_{r}, L_{r}$, and $B_{Q_{r}}$ in $\mathbb{C}_{r}$.

The sufficient regulation condition (4.18) given in Theorem 4.3 is equivalent to

$$
\beta<\delta_{0}-\Delta \beta_{m}-\varepsilon
$$

Using $\beta=c w_{m} / \alpha$ and letting $\kappa=\left(\delta_{0}-\Delta \beta_{m}-\varepsilon\right) / w_{m}$, condition (4.18) is equivalent to

$$
\frac{c}{\alpha}<\kappa
$$

If (5.2) cannot be satisfied, the switching surface $S$ intersects the set $B_{\gamma}$ and conditions (4.19) and (4.20) in Theorem 4.4 need to be verified. For the switched closed loop system (3.7), the parameters $c$ and $\alpha$ can be estimated using the following matrix inequalities [22]:

$$
\begin{gathered}
\widehat{A}_{r}^{T} P+P \widehat{A}_{r}+2 \alpha P<0, \quad \alpha>0, r \in\{1,2\}, \\
I<P<c^{2} I, \quad c>0 .
\end{gathered}
$$


If the matrices $K_{r}, L_{r}, B_{Q_{r}}$, and $C_{Q_{r}}$ in $\widehat{A}_{r}$ are unknown, then the above equations define a bilinear matrix inequality. Combining (5.2) and (5.3), the synthesis procedure for the controller $\mathbb{C}_{r}$ can be realized by solving the following BMIs:

$$
\begin{gathered}
{\left[\begin{array}{ccc}
A_{r}+B_{r} K_{r} & B_{r} K_{r} & B_{r} \Psi_{r}-B_{r} K_{r} \Omega_{r} \\
0 & A_{r}+L_{r} C_{r}^{y} & D_{r}^{x}+L_{r} D_{r}^{y} \\
0 & B_{Q_{r}} C_{r}^{y} & H_{r}+B_{Q_{r}} D_{r}^{y}
\end{array}\right]^{T} P} \\
+P\left[\begin{array}{ccc}
A_{r}+B_{r} K_{r} & B_{r} K_{r} & B_{r} \Psi_{r}-B_{r} K_{r} \Omega_{r} \\
0 & A_{r}+L_{r} C_{r}^{y} & D_{r}^{x}+L_{r} D_{r}^{y} \\
0 & B_{Q_{r}} C_{r}^{y} & H_{r}+B_{Q_{r}} D_{r}^{y}
\end{array}\right]+2 \alpha P<0, \quad \alpha>0, r \in\{1,2\} \\
I<P<c^{2} I, \quad c>0 \\
\frac{c}{\alpha}<\kappa,
\end{gathered}
$$

where the unknown parameters are $K_{r}, L_{r}, B_{Q_{r}}, P, \alpha$, and $c$. Once $K_{r}, L_{r}, B_{Q_{r}}$ are determined based on the above BMIs, the matrices $A_{Q_{r}}$ and $C_{Q_{r}}$ can be calculated using (4.3) as given in Theorem 4.2.

In this paper, a regulator synthesis algorithm, referred to as the $P$-C iteration algorithm, will be used to find a parameterized regulator that satisfies either one of the regulation conditions (4.18), (4.19), or (4.20). The $P-\mathbb{C}$ algorithm iteratively solves for the Lyapunov matrix $P$ and the parameters in the controller $\mathbb{C}_{r}$ given in (2.3). The basic idea of the $P$ - $\mathbb{C}$ iteration is that a BMI can be converted into an LMI when some of the parameters in the BMI are fixed. The approach for solving BMI problems is to alternate between two optimization problems subject to LMIs, which are related to the matrix $P$ in (5.4) and (5.5) and the parameters in thecontroller $\mathbb{C}_{r}$ in (2.3), respectively. In the algorithm, the input data is represented by $A_{r}, B_{r}, C_{r}^{y}, C^{e}, D_{r}^{x}$, $D_{r}^{y}, D_{r}^{e}, H_{r}, \delta$, and $w_{m}$, whereas the unknown variables to be determined are $K_{r}, L_{r}, B_{Q_{r}}, A_{Q_{r}}$, $C_{Q_{r}}, P, \alpha$, and $c$. The algorithm is summarized below, where $K_{r}^{i}, L_{r}^{i}, B_{Q_{r}}^{i}, P_{i}, \alpha_{i}$, and $c_{i}$ denote the solutions $K_{r}, L_{r}, B_{Q_{r}}, P, \alpha$, and $c$ obtained at the $i$ th iteration of the algorithm.

(1) Calculating $\kappa$ : determine $\left(\Omega_{r}, \Psi_{r}\right)$ by solving the Sylvester equation (4.2) and let $\Pi_{r}=$ $\left[\begin{array}{c}\Omega_{r} \\ \Phi_{r}\end{array}\right]$ with $\Phi_{r}=\left[\begin{array}{c}\Omega_{r} \\ i\end{array}\right]$. Let $\widehat{\Pi}_{r}$ be as in (4.10). Determine $\Delta \beta_{m}$ using (4.16), then calculate $\mathcal{\kappa}=\left(\delta_{0}-\Delta \beta_{m}-\varepsilon\right) / w_{m}$.

(2) Initializing $\mathbb{C}_{r}$ : initialize the controller parameters $K_{r}^{0}, L_{r}^{0}$, and $B_{O_{r}}^{0}$ to make the switched closed loop system (3.7) internally stable, which can be realized by solving the following two LMIs separately for the unknown matrices $K_{r}, L_{r}, B_{Q_{r}}, P_{K}$, and $P_{L}$ with preset constants $\alpha_{K} \geq 0$ and $\alpha_{L} \geq 0$,

$$
\begin{gathered}
{\left[A_{r}+B_{r} K_{r}\right]^{T} P_{K}+P_{K}\left[A_{r}+B_{r} K_{r}\right]+\alpha_{K} P_{K}<0, \quad r \in\{1,2\},} \\
{\left[\begin{array}{cc}
A_{r}+L_{r} C_{r}^{y} & D_{r}^{x}+L_{r} D_{r}^{y} \\
B_{Q_{r}} C_{r}^{y} & H_{r}+B_{Q_{r}} D_{r}^{y}
\end{array}\right]^{T} P_{L}+P_{L}\left[\begin{array}{cc}
A_{r}+L_{r} C_{r}^{y} & D_{r}^{x}+L_{r} D_{r}^{y} \\
B_{Q_{r}} C_{r}^{y} & H_{r}+B_{Q_{r}} D_{r}^{y}
\end{array}\right]+\alpha_{L} P_{L}<0, \quad r \in\{1,2\} .}
\end{gathered}
$$


(3) Initializing $\alpha_{0}$ : based on the initial controller parameters $K_{r}^{0}, L_{r}^{0}$, and $B_{Q_{r}}^{0}$ from step 2, initialize the maximum decay rate $\alpha_{0}$ by solving the following optimization problem:

$\max \alpha$

subject to (5.4).

(4) P-Step: At the $i$ th iteration, $i=1,2, \ldots$, given the matrices $K_{r}^{i-1}, L_{r}^{i-1}$, and $B_{Q_{r}}^{i-1}$ and the scalar $\alpha_{i-1}$, solve the following optimization problem for $P_{i}$ and $c_{i}$ :

$\min c$

subject to (5.4) and (5.5).

(5) $\mathbb{C}$-Step: At the $i$ th iteration, $i=1,2, \ldots$, given the matrix $P_{i}$ and $c_{i}$ from step 4 , solve the following optimization problem for $K_{r}^{i}, L_{r}^{i}, B_{Q_{r^{\prime}}}^{i}$, and $\alpha_{i}$ :

$\max \alpha$

subject to (5.4) and (5.5).

(6) Verification: Verify the constraints (5.6), (4.19), and (4.20), and if any of the three conditions is satisfied, compute $A_{Q_{r}}$ and $C_{Q_{r}}$ using (4.3), then stop the algorithm. If none of (5.6), (4.19), and (4.20) is satisfied, then go to step 4.

The iterative loop is repeatedly executed until a solution is found, or there is no major reduction in $c / \alpha$ relative to the previous iteration. The algorithm converges to a local solution of $c / \alpha$, since at each $i$ th iteration of the algorithm, we have $c_{i} \leq c_{i-1}$ and $\alpha_{i} \geq \alpha_{i-1}$. Therefore, if no solution can satisfy the regulation conditions (5.6), (4.19), or (4.20) by the iterative procedure described above, then the initial parameters $K_{r}^{0}, L_{r}^{0}$, and $B_{Q_{r}}^{0}$ can be adjusted by changing the decay rates $\alpha_{K}$ and $\alpha_{L}$ in (5.7), and restarting the iterative procedure again. If the above algorithm yields a solution, then, according to Theorems 4.3 or 4.4 , the switched closed loop system will achieve regulation.

\section{Numerical example}

In this section, the regulator synthesis method proposed in this paper will be used to design a controller that cancels the contact vibrations in a mechanical system. Figure 4 shows the diagram of such a system consisting of a mass $m$, a contact surface $S_{c}$, and their respective coordinates. The mass $m$ is attached to a spring with stiffness $k$ and a damper with damping coefficient $c$. The mass $m$ moves only in the vertical direction, whereas the contact surface underneath it moves to the left. The mass $m$ may enter into intermittent contact with the surface $S_{c}$, resulting in contact vibrations. When the mass $m$ enters into contact with the surface $S_{c}$, the contact characteristics are represented by a spring with stiffness $k_{c}$ and a damper with damping coefficient $c_{c}$. The force $F$ represents the external force used to control the mass $m$, whereas $F_{a}$ represents a disturbance force. This model can be found in many applications, such as the interface between the read/write head and the disk surface in hard disk drive systems. In the following, and with respect to the system shown in Figure 4, 


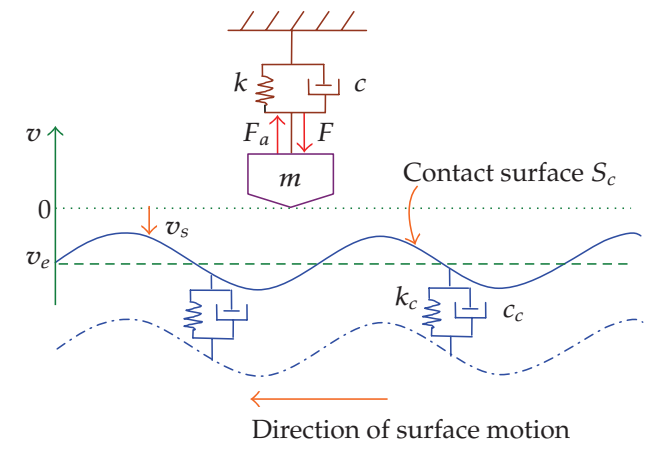

Figure 4: Diagram of a mechanical system with switched dynamics.

the control objective is for the mass $m$ to follow the displacement of the contact surface $S_{c}$, while maintaining a desired constant separation in the vertical direction. Let $v$ be the deviation of the mass from its equilibrium position. Then the equations of motion of the mass can be written as

$$
\begin{aligned}
& m \ddot{v}+c \dot{v}+k v=-F+F_{a}, \quad \text { when } v>v_{s}(\text { noncontact mode), } \\
& m \ddot{v}+c \dot{v}+k v=-F+F_{a}+F_{c}, \quad \text { when } v \leq v_{s}(\text { contact mode), }
\end{aligned}
$$

where $F_{c}$ is the contact force expressed as

$$
F_{c}=c_{c}\left(\dot{v}_{s}-\dot{v}\right)+k_{c}\left(v_{s}-v\right)
$$

and where $v_{s}$ is the displacement of the contact surface. The wavy contact surface profile is expressed as a linear combination of sinusoidal functions:

$$
v_{s}=\sum_{k} c_{k} \cos \left(\omega_{k} t+\phi_{k}\right)+v_{e}
$$

with amplitudes $c_{k}$, frequencies $\omega_{k}$, phases $\phi_{k}, k=1,2, \ldots$, and constant offset $v_{e}$. Therefore, the distance between the mass $m$ and contact surface $S_{c}$ is $v-v_{s}$. The control signal is defined as $u=-F / m$ for the noncontact situation and $u=-\left(F-k_{c} v_{e}\right) / m$, when contact takes place. Let $-v_{e}$ denote the desired distance between the mass $m$ and contact surface $S_{c}$. The output $y$, to be fed to the controller, is defined as

$$
y=v
$$

The performance variable $e$ is defined to be the difference between the actual distance $v-v_{S}$ and the desired distance $-v_{e}$,

$$
e=v-v_{s}+v_{e}
$$


Therefore, the system will switch between the contact and noncontact modes according to the value of the performance variable $e$. If $e>\delta=v_{e}$, the system will operate in the noncontact mode, and if $e \leq \delta=v_{e}$, the system will operate in the contact mode. Let $x_{1}=v$ and $x_{2}=\dot{v}$, then the switched system model is given by

$$
\Sigma_{r}:\left\{\begin{array}{l}
{\left[\begin{array}{l}
\dot{x}_{1} \\
\dot{x}_{2}
\end{array}\right]=A_{r}\left[\begin{array}{l}
x_{1} \\
x_{2}
\end{array}\right]+\left[\begin{array}{l}
0 \\
1
\end{array}\right] u+\left[\begin{array}{ll}
0 & 0 \\
1 & 0
\end{array}\right] d_{r}} \\
y=\left[\begin{array}{ll}
1 & 0
\end{array}\right]\left[\begin{array}{l}
x_{1} \\
x_{2}
\end{array}\right] \\
e=\left[\begin{array}{ll}
1 & 0
\end{array}\right]\left[\begin{array}{l}
x_{1} \\
x_{2}
\end{array}\right]+\left[\begin{array}{ll}
0 & -1
\end{array}\right] d_{r} \\
r=\left\{\begin{array}{l}
1 \\
\text { if } e \leq v_{e}, \\
2 \text { if } e>v_{e},
\end{array}\right.
\end{array}\right.
$$

where

$$
\begin{aligned}
& A_{1}=\left[\begin{array}{cc}
0 & 1 \\
-\frac{\left(k+k_{c}\right)}{m} & -\frac{\left(c+c_{c}\right)}{m}
\end{array}\right], \\
& A_{2}=\left[\begin{array}{cc}
0 & 1 \\
-\frac{k}{m} & -\frac{c}{m}
\end{array}\right], \\
& d_{1}=\left[\begin{array}{c}
\frac{1}{m}\left(c_{c} \dot{v}_{s}+k_{c} v_{s}-k_{c} v_{e}+F_{a}\right) \\
v_{s}-v_{e}
\end{array}\right], \\
& d_{2}=\left[\begin{array}{c}
F_{a} / m \\
v_{s}-v_{e}
\end{array}\right] .
\end{aligned}
$$

Consider a model for the read/write head and disk surface interface in hard disk drive systems similar to that in $[11,12]$. The surface profile is given by $v_{s}=(\sin (120 \pi t)-30) \times 10^{-6} \mathrm{~m}$, and the force $F_{a}=(1.5 \sin (120 \pi t+\pi)+0.1 \cos (120 \pi t+\pi)) \times 10^{-3} \mathrm{~N}$. Therefore, we have $\delta=v_{e}=-30 \times 10^{-6} \mathrm{~m}$. Let $\mathrm{m}, k, c, k_{c}$, and $c_{c}$ be $200 \mathrm{mg}, 1.5 \times 10^{3} \mathrm{~N} / \mathrm{m}, 0.05 \mathrm{~N} / \mathrm{m} / \mathrm{Sec}$, $1.5 \times 10^{4} \mathrm{~N} / \mathrm{m}$, and $0.3 \mathrm{~N} / \mathrm{m} / \mathrm{Sec}$, respectively. In the following, a regulator for the switched system is designed using the synthesis procedure described in the previous section. Simulation results will illustrate the performance of the proposed regulator in maintaining the desired system output despite the presence of switching. 
In order to find a feasible solution for the BMIs, we introduce a coordinate transformation as $\left[\begin{array}{l}x_{1}^{\prime} \\ x_{2}^{\prime}\end{array}\right]=T\left[\begin{array}{l}x_{1} \\ x_{2}\end{array}\right]$, where $T=\left[\begin{array}{cc}0.25 & 0.25 \times 10^{-8} \\ 0.50 & -0.50 \times 10^{-8}\end{array}\right]$. Rewriting (6.6) in the state space form (2.1) results in

$$
\begin{aligned}
& A_{1}=\left[\begin{array}{cc}
49999991 & -24999995 \\
100000017 & -50000008
\end{array}\right], \\
& A_{2}=\left[\begin{array}{rr}
49999998 & -24999999 \\
100000002 & -50000001
\end{array}\right] \text {, } \\
& H_{1}=H_{2}=\left[\begin{array}{cc}
0 & 120 \pi \\
-120 \pi & 0
\end{array}\right], \quad w_{10}=w_{20}=\left[\begin{array}{c}
0 \\
1 \times 10^{-6}
\end{array}\right], \quad B_{1}=B_{2}=\left[\begin{array}{c}
0.25 \times 10^{-8} \\
-0.50 \times 10^{-8}
\end{array}\right] \text {, } \\
& C_{1}^{y}=C_{2}^{y}=C^{e}=\left[\begin{array}{ll}
2 & 1
\end{array}\right], \\
& D_{1}^{x}=\left[\begin{array}{cc}
0.21 & 1.41 \times 10^{-3} \\
-0.41 & -2.82 \times 10^{-3}
\end{array}\right], \quad D_{2}^{x}=\left[\begin{array}{cc}
1.87 \times 10^{-2} & 1.25 \times 10^{-3} \\
-3.75 \times 10^{-2} & -2.50 \times 10^{-3}
\end{array}\right], \quad D_{1}^{y}=D_{2}^{y}=0, \\
& D_{1}^{e}=D_{2}^{e}=\left[\begin{array}{ll}
-1 & 0
\end{array}\right] .
\end{aligned}
$$

It is desired to design a regulator that can reject the disturbance in the switched system. Based on Theorem 4.2, solving the Sylvester equation (4.2) yields

$$
\begin{gathered}
\Psi_{1}=\left[\begin{array}{ll}
-14.21 & 9.42
\end{array}\right] \times 10^{4}, \quad \Psi_{2}=\left[\begin{array}{ll}
-14.21 & -40.58
\end{array}\right] \times 10^{4}, \\
\Omega_{1}=\Omega_{2}=\left[\begin{array}{cc}
0.25 & 9.42 \times 10^{-7} \\
0.50 & -1.88 \times 10^{-6}
\end{array}\right], \quad \Pi_{1}=\Pi_{2}=\left[\begin{array}{c}
\Omega_{1} \\
\Omega_{1} \\
I_{2 \times 2}
\end{array}\right] .
\end{gathered}
$$

Based on (4.16), we obtain $\Delta \beta_{m}=1.76 \times 10^{-6}$. Since $\delta=-30 \times 10^{-6} \mathrm{~m}$ and $w_{m}=0.62 \times 10^{-6}$, and with $\varepsilon=0.01$, we have $\kappa=18.81$ in (5.2). Using the $P$ - $\mathbb{C}$ algorithm proposed above, we obtain

$$
\begin{aligned}
K_{1} & =\left[\begin{array}{ll}
-4.201 .88
\end{array}\right] \times 10^{15}, & K_{2} & =\left[\begin{array}{ll}
-8.98 & 4.02
\end{array}\right] \times 10^{15}, \\
L_{1} & =\left[\begin{array}{l}
-1.51 \\
-3.02
\end{array}\right] \times 10^{7}, & L_{2} & =\left[\begin{array}{c}
-0.52 \\
-1.04
\end{array}\right] \times 10^{7}, \\
B_{Q_{1}} & =\left[\begin{array}{c}
-29.69 \\
4.34
\end{array}\right] \times 10^{7}, & B_{Q_{2}} & =\left[\begin{array}{c}
-5.21 \\
3.91
\end{array}\right] \times 10^{7}, \quad \frac{c}{\alpha}=23.61 .
\end{aligned}
$$

Then, based on (4.3), we have

$$
C_{Q_{1}}=[11006.437 .51] \times 10^{4}, \quad C_{Q_{2}}=[23532.541 .63] \times 10^{4} .
$$




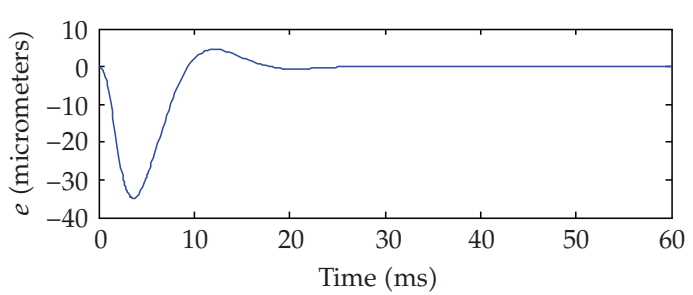

(a)

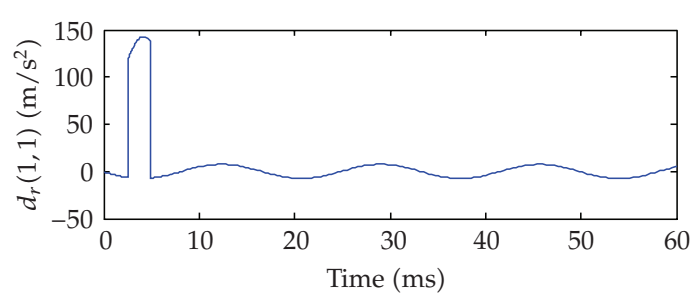

(b)

Figure 5: Simulation results for the case of $v_{e}=-30$ micrometers showing the performance variable $e$ and the switching component $d_{r}(1,1)$ in the disturbance $d_{r}$.

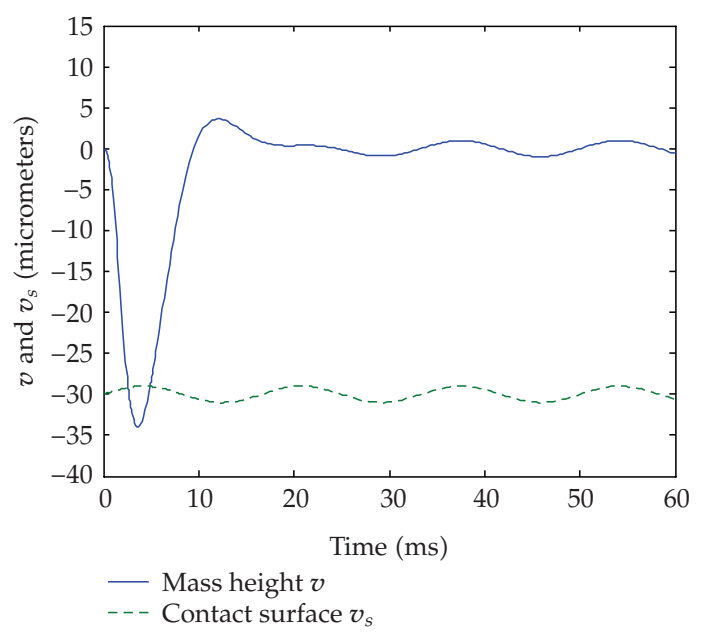

Figure 6: Simulation results for the case of $v_{e}=-30$ micrometers showing the regulated mass height $v$ and the contact surface displacement $v_{s}$.

Since $c / \alpha=23.61>\kappa=18.81$, condition (5.6) is not satisfied. Then conditions (4.19) and (4.20) will be verified. First, we obtain $\gamma=c w_{m} / \alpha+\Delta \beta_{m}+\varepsilon=16.41 \times 10^{-6}$. Hence, after a long enough time, the trajectory will enter into the bounded set $B_{\gamma}$ with $\gamma=16.41 \times 10^{-6}$ and continue to evolve thereafter. Given that $\delta<0$ and that

$$
\begin{gathered}
C^{e x}\left(C^{e x} \widehat{A}_{1}\right)^{T}=3 \times 10^{8}>0, \\
b^{T}\left(A_{s 1} A_{s 1}^{T}\right)^{-1} b=281.25 \times 10^{-12}>r^{2}=268.91 \times 10^{-12},
\end{gathered}
$$

it follows that regulation condition (4.20) in Theorem 4.4 is satisfied. Therefore, regulation can be achieved in the switched closed loop system using the designed controller.

The simulation results of the response of the closed loop system under switching are illustrated in Figures (5-6). It can be seen that if the performance variable is smaller than -30 micrometers, the mass enters into contact with the contact surface $S_{c}$ and the model of the system switches. It can also be seen that the disturbance $d_{r}$ changes at the switching times. But even in the presence of switching, the switched system performance variable $e$ still converges to zero, which means the mass $m$ asymptotically follows the contact surface $S_{c}$ at the desired separation 30 micrometers. 


\section{Conclusion}

The problem of regulation in switched bimodal systems against known disturbance or reference signals is discussed. A regulator design approach based on the parameterization of a set of controllers that can achieve regulation for the switched system is presented. The forced switched closed loop system involving the parameterized controller is transformed into an unforced impulsive switched system. Consequently, the original regulation problem is transformed into a stability analysis problem for the origin of the impulsive switched system. Sufficient conditions for regulation and a regulator synthesis method based on solving a set of bilinear matrix inequalities are presented. A simulation example is used to illustrate the effectiveness of the proposed regulator.

\section{Acknowledgments}

The authors would like to thank the associate editor and the anonymous reviewers for their comments. This research was supported by the Natural Sciences and Engineering Research Council of Canada and the provincial government of Ontario.

\section{References}

[1] D. Cheng, L. Guo, Y. Lin, and Y. Wang, "Stabilization of switched linear systems," IEEE Transactions on Automatic Control, vol. 50, no. 5, pp. 661-666, 2005.

[2] V. D. Blondel and J. N. Tsitsiklis, "Complexity of stability and controllability of elementary hybrid systems," Automatica, vol. 35, no. 3, pp. 479-489, 1999.

[3] T. Ooba and Y. Funahashi, "On a common quadratic Lyapunov function for widely distant systems," IEEE Transactions on Automatic Control, vol. 42, no. 12, pp. 1697-1699, 1997.

[4] R. Shorten, K. S. Narendra, and O. Mason, "A result on common quadratic Lyapunov functions," IEEE Transactions on Automatic Control, vol. 48, no. 1, pp. 110-113, 2003.

[5] M. S. Branicky, "Multiple Lyapunov functions and other analysis tools for switched and hybrid systems," IEEE Transactions on Automatic Control, vol. 43, no. 4, pp. 475-482, 1998.

[6] M. Johansson and A. Rantzer, "Computation of piecewise quadratic Lyapunov functions for hybrid systems," IEEE Transactions on Automatic Control, vol. 43, no. 4, pp. 555-559, 1998.

[7] J. M. Gonçalves, A. Megretski, and M. A. Dahleh, "Global analysis of piecewise linear systems using impact maps and surface Lyapunov functions," IEEE Transactions on Automatic Control, vol. 48, no. 12, pp. 2089-2106, 2003.

[8] D. Liberzon, Switching in Systems and Control, Systems \& Control: Foundations \& Applications, Birkhäuser, Boston, Mass, USA, 2003.

[9] Z. Sun and S. S. Ge, Switched Linear Systems. Control and Design, Springer, London, UK, 2005.

[10] E. K. Boukas, Stochastic Switching Systems: Analysis and Design, Control Engineering, Birkhäuser, Boston, Mass, USA, 2006.

[11] K. Linda, K. Ono, and M. Yamane, "Dynamic characteristics and design consideration of a tripad slider in the near-contact regime," ASME Journal of Tribology, vol. 124, no. 3, pp. 600-606, 2002.

[12] Z. Wu and F. Ben Amara, "Adaptive regulation of the flying height for 2-DOF tripad slider in hard disk drives," in Proceedings of the IEEE International Conference on Mechatronics and Automation, vol. 1, pp. 160-165, Niagara Falls, Ontario, Canada, July-August 2005.

[13] E. J. Davison, "The robust control of a servomechanism problem for linear time-invariant multivariable systems," IEEE Transactions on Automatic Control, vol. 21, no. 1, pp. 25-34, 1976.

[14] B. A. Francis and W. M. Wonham, "The internal model principle of control theory," Automatica, vol. 12, no. 5 , pp. 457-465, 1976.

[15] B. A. Francis, "The linear multivariable regulator problem," SIAM Journal on Control and Optimization, vol. 15, no. 3, pp. 486-505, 1977.

[16] A. Isidori and C. I. Byrnes, "Output regulation of nonlinear systems," IEEE Transactions on Automatic Control, vol. 35, no. 2, pp. 131-140, 1990. 
[17] C. I. Byrnes, F. D. Priscoli, A. Isidori, and W. Kang, "Structurally stable output regulation of nonlinear systems," Automatica, vol. 33, no. 3, pp. 369-385, 1997.

[18] S. Devasia, B. Paden, and C. Rossi, "Exact-output tracking theory for systems with parameter jumps," International Journal of Control, vol. 67, no. 1, pp. 117-131, 1997.

[19] K. Sakurama and T. Sugie, "Trajectory tracking control of bimodal piecewise affine systems," International Journal of Control, vol. 78, no. 16, pp. 1314-1326, 2005.

[20] K. Zhou, J. C. Doyle, and K. Glover, Robust and Optimal Control, Prentice Hall, Upper Saddle River, NJ, USA, 1996.

[21] F. Ben Amara, P. T. Kabamba, and A. G. Ulsoy, "Adaptive sinusoidal disturbance rejection in linear discrete-time systems_-part I: theory," Journal of Dynamic Systems, Measurement, and Control, vol. 121, no. 4, pp. 648-654, 1999.

[22] S. Boyd, L. El Ghaoui, E. Feron, and V. Balakrishnan, Linear Matrix Inequalities in System and Control Theory, vol. 15 of SIAM Studies in Applied Mathematics, Society for Industrial and Applied Mathematics, Philadelphia, Pa, USA, 1994. 


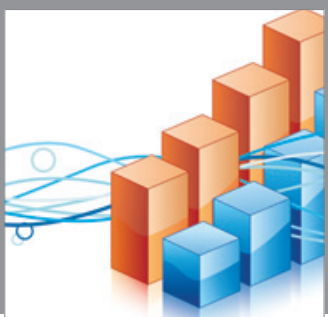

Advances in

Operations Research

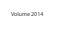

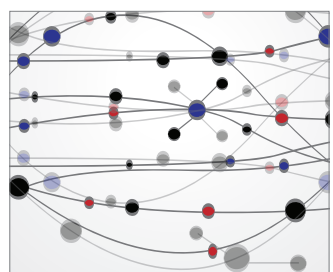

\section{The Scientific} World Journal
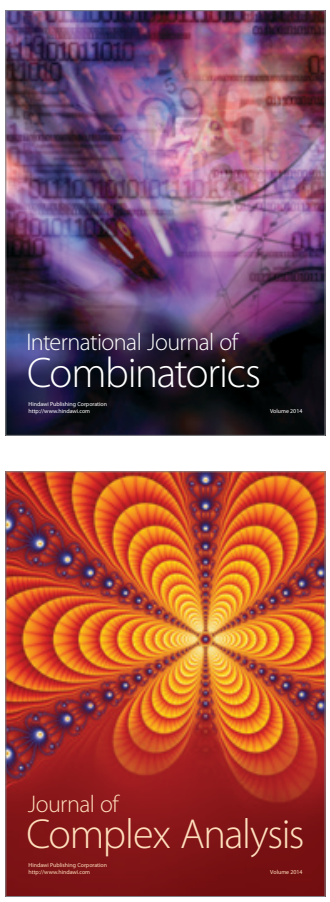

International Journal of

Mathematics and

Mathematical

Sciences
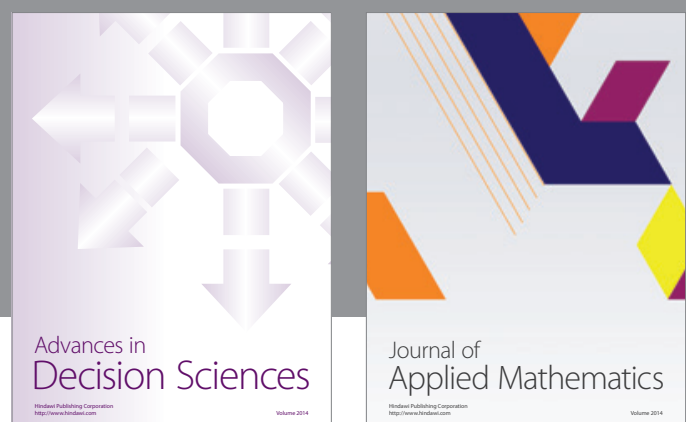

Journal of

Applied Mathematics
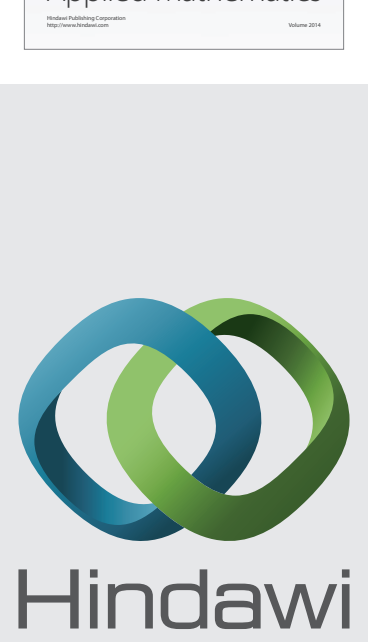

Submit your manuscripts at http://www.hindawi.com
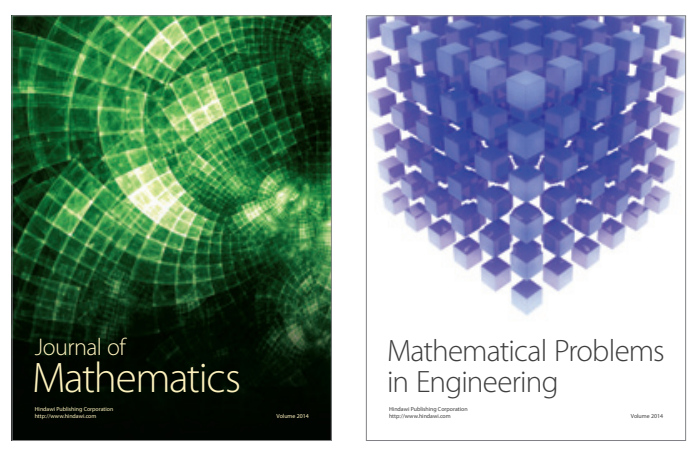

Mathematical Problems in Engineering
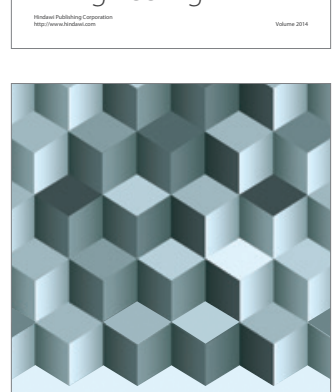

Journal of

Function Spaces
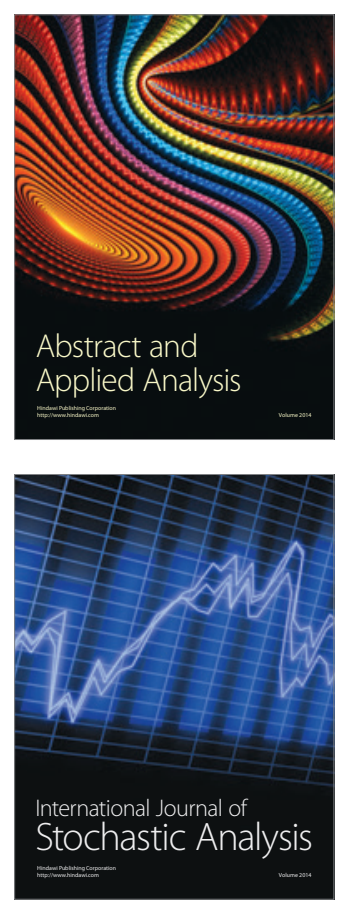

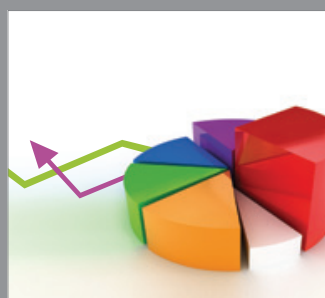

ournal of

Probability and Statistics

Promensencen
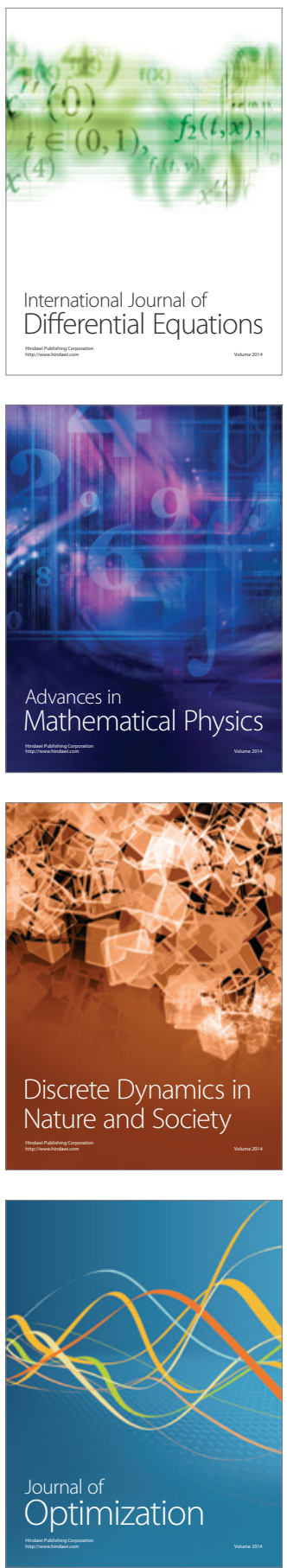